\title{
Invited Speaker
}

\section{Talking about the world with a distributed model}

\author{
Gemma Boleda \\ Universitat Pompeu Fabra
}

\begin{abstract}
We use language to talk about the world, and so reference is a crucial property of language. However, modeling reference is particularly difficult, as it involves both continuous and discrete aspects of language. For instance, referring expressions like "the big mug" or "it" typically contain content words ("big", "mug"), which are notoriously fuzzy or vague in their meaning, and also function words ("the", "it") that largely serve as discrete pointers. Data-driven, distributed models based on distributional semantics or deep learning excel at the former, but struggle with the latter, and the reverse is true for symbolic models. I present ongoing work on modeling reference with a distributed model aimed at capturing both aspects, and learns to refer directly from reference acts.
\end{abstract}

